Psychiatrische Notfälle

\title{
Patient rastet aus - was tun?
}

\author{
Auch als Hausarzt werden Sie gelegentlich mit psychiatrischen Notfallsituationen \\ konfrontiert, die rasches medikamentöses Handeln erfordern. Welche Substanz \\ ist wann am effektivsten und welche Risiken sollten Sie beachten?
}

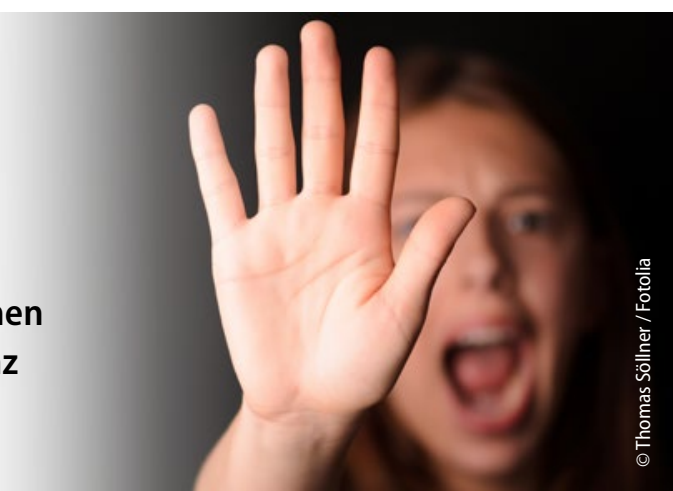

_ Die Häufigkeit psychiatrischer Notfälle hat in den letzten zehn Jahren deutlich zugenommen. Dabei handelt es sich meist um nicht organische psychische Störungen, es sollten aber auch immer organische (z.B. nach Schlaganfall oder Hypoglykämie) und medikamentöse Ursachen differenzialdiagnostisch in Erwägung gezogen werden.

$\mathrm{Zu}$ den häufigsten psychiatrischen Notfallsituationen zählen Intoxikationen, Suizidalität, Angst- und Erregungszustände sowie das Delir, welches durch die Leitsymptome Bewusstseinsstörungen, kognitive Störungen, Desorientiertheit, optische Halluzinationen, psychomotorische Unruhe und Suggestibilität gekennzeichnet ist. Dazu kommen Ataxie, Dysarthrie, Tremor und vegetative Symptome.

\section{Keine spezifische Notfallmedikation}

„Der Vielfalt der psychiatrischen Diagnosen steht eine nur geringe Anzahl notfallpsychiatrisch relevanter Syndrome gegenüber, es gibt daher keine krankheitsspezifischen Notfallmedikamente“, erläuterte Prof. Thomas Messer, Pfaffenhofen. Ziel der Notfalltherapie ist also nicht die Behandlung der zugrunde liegenden Erkrankung, sondern die Kontrolle des Symptoms und die Wiederherstellung eines adäquaten Arzt-Patienten-Kontakts. Dem behandelnden Arzt sollte dabei immer die Wirkungsintensität, das Nebenwirkungsprofil und das Interaktionsverhalten des eingesetzten Medikaments bekannt sein. Die Substanz sollte schnell applizierbar und schnell wirksam sein.
Welche Substanz gegen welche Krise? Antipsychotika . Ein in der Notfallpsychiatrie häufig verwendetes Präparat ist Haloperidol, welches bei Bedarf mit Promethazin i.m. oder Risperidon kombiniert werden kann. Bei geriatrischen $\mathrm{Pa}$ tienten oder solchen mit internistischen Erkrankungen haben sich besonders Melperon und Pipamperon bewährt. Bei agitierten Patienten im Rahmen einer Schizophrenie oder Manie empfiehlt sich die i.m.Gabe eines Antipsychotikums der 2. Generation wie Olanzapin, Aripiprazol oder Ziprasidon. „Trizyklische niederpotente Neuroleptika wie Levomepromazin sollten wegen ihrer anticholinergen und kardiovaskulären $\mathrm{Ne}$ benwirkungen im akuten

Erregungszustand nicht mehr eingesetzt werden", so Messer.

Sedativa - Als Sedativum und Anxiolytikum hat sich das relativ kurz wirksame Benzodiazepin Lorazepam bewährt. Es wirkt zusätzlich antikonvulsiv, hypnotisch und muskelrelaxierend. Es kann bei besonders starker Agitiertheit mit Haloperidol oder Risperidon kombiniert werden. Bei prädeliranten bzw. deliranten Patienten ist das sedierende Clomethiazol empfehlenswert, das jedoch nur über eine sehr geringe therapeutische Breite verfügt. Midazolam wird für psychiatrische Notfälle dagegen nicht empfohlen.

Applikationsformen · Bei einer parenteralen Applikation muss die Dosis reduziert werden, i.v.-Injektionen müssen immer sehr langsam erfolgen, und Haloperidol sollte nur oral oder i.m. gegeben werden, da bei i.v.-Gabe gefährliche ven- trikuläre Tachykardien als Folge einer QT-Zeit-Verlängerung auftreten können.

\section{Risiken der psychiatrischen Notfallmedikation}

Nach der Gabe eines Antipsychotikums kann es zu extrapyramidalmotorischen Störungen kommen, die sich in Hyperkinesen der mimischen Muskulatur, Blickkrämpfen und Torticollis als Folge einer Hyperaktivität der cholinergen Transmission äußern können. Therapie der Wahl sind zentral wirksame Anticholinergika wie Biperiden.

Etwa zwei Wochen nach Beginn einer Therapie mit einem Antipsychotikum kann sich, als Folge einer Blockade der Dopamin-Rezeptoren, ein malignes neuroleptisches Syndrom entwickeln. Die Klinik ist durch Fieber, Tremor, Bewusstseinsstörungen, Tachykardie und Dyskinesien gekennzeichnet. Therapeutisch empfehlen sich Kühlung, Flüssigkeits- und Elektrolytsubstitution und die Gabe von Dopaminergika.

Das Serotonin-Syndrom entsteht durch Interaktionen serotonin-potenzierender Substanzen, wenn z.B. ein SSRI mit Kokain, Tramadol, Fentanyl oder Triptanen kombiniert wird. Klinisch imponieren Tremor, Muskeltonuserhöhung und Kloni. In der Akutsituation sollte Cyproheptadin oral gegeben werden.

Dr. Peter Stiefelhagen

- Quelle: DGPPN-Kongress 2015 in Berlin

Mehr zum Thema

Pharmakotherapie von psychiatrischen Akut- und Notfallsituationen

$\rightarrow$ www.springermedizin.de/5840250 